\title{
The Klein Bottle: Variations on a Theme
}

\section{Gregorio Franzoni}

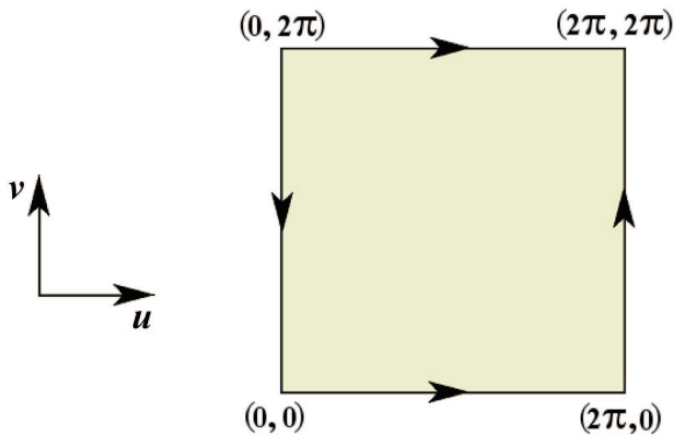

Figure 1. The Klein bottle as a square with the opposite sides identified in the sense of the arrows.

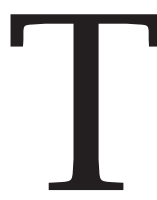

he Klein bottle ( $\mathbb{K}$ in the following) is a topological object that can be defined as the closed square $[0,2 \pi] \times[0,2 \pi]$ with the opposite sides identified according to the equivalence relation

$$
\begin{aligned}
& (u, 0) \sim(u, 2 \pi), \\
& (0, v) \sim(2 \pi, 2 \pi-v) .
\end{aligned}
$$

It is a well-known fact that $\mathbb{K}$ is a genus 2 nonorientable closed surface with Euler characteristic $x=0$, which is topologically equivalent to a couple of Möbius bands glued together along the border, and that it is nonembeddable in $\mathbb{R}^{3}$. It is possible, however, to immerse it in $\mathbb{R}^{3}$, that is, to map it into $\mathbb{R}^{3}$ obtaining an image with no singular points. To give an immersion of $\mathbb{K}$ in $\mathbb{R}^{3}$, it suffices to define, on the fundamental square $[0,2 \pi] \times[0,2 \pi]$, an immersion that passes to the quotient with respect to the relation $\sim$. Felix Klein, in his original work in 1882 [18, §23],

Gregorio Franzoni teaches mathematics in secondary school and at the University of Cagliari. His email address is gregoriofranzoni@gmai 1 . com.

DOI: http://dx.doi.org/10.1090/noti880
[15, pp. 308-310], described the object as follows: tucking a rubber hose, making it penetrate itself, and then smoothly gluing the two ends together, but he does not give any equation. Today we have some equations for this topological object which are fully satisfactory from a technical point of view and that can be called canonical for their simplicity and because of the clear and understandable shapes they lead to. They are due to T. Banchoff and B. Lawson and are described in detail later in this article. However, they do not resemble the object arising from the geometrical construction given by Klein, which will be called the classical shape in the following. It would also be desirable to have a good parametrization, with simple formulas and a nice shape, for the bottle in its classical version. Of course, the newer shapes are topologically equivalent to the classical one, but as immersions they belong to different regular homotopy classes (see the section about regular homotopy classes of immersed surfaces), so it makes sense to find a canonical expression for the classical bottle too, apart from historical and aesthetic reasons. To elaborate, we say that when a mathematical subject has a missing tessera, it is often perceived as a challenge to researchers, who put their best effort into it and sometimes go far beyond the original target, opening new research threads and/or revealing unknown links between different fields. The well-known story of the Costa surface and of the associated new family of minimal surfaces discovered by D. Hoffman and W. H. Meeks and the sphere eversion story, both summarized by R. S. Palais [20], on the one hand have challenged and inspired a number of geometers. On the other hand, they have played a key role in the development of mathematical visualization techniques and have contributed to bringing to the public's attention beautiful and spectacular aspects of mathematics. Finding good 
mathematical expression for surfaces is also useful if one wants to realize material models of them. Wire models of the Boy surface and of halfway steps of a sphere eversion made by F. Apéry and later by myself [2], [7] could be done because Apéry had expressed those surfaces as families of simple curves in space [1]. To say something more about material models of surfaces, we can claim that today the greatest potential in the field lies in $3 D$ printing systems, which help bringing 3D computer-generated models to the real world and that are today much more affordable than only a decade ago (less than US\$10,000 for an entry-level system).

Tactile representation of mathematical objects is a natural extension of their visualization and, similarly, is a great source of inspiration for both young and senior mathematicians and an excellent way to highlight or communicate properties and concepts such as curvature, minimality, orientability, geodesic curves, singularity, and so on. Some very interesting work in this field has been done by pioneers like S. Dickson (see for example [7], [12]) and C. Séquin [22]. If the famous Brill-Schilling collection of plaster and wire models [13] had to be stopped in the 1920s because of high production costs and lack of interest by mathematicians, whose attention moved towards formalism and abstraction rather than visual and intuitive aspects, today we see a renewed interest in images and models. The trend portrayed by Palais [20] more than a decade ago is now stronger than ever, nourished also by a great interest in artistic expression inspired by mathematics. I believe that the time for the production of an extensive collection of models of surfaces, improved and updated if compared to Schilling's, might have arrived. In fact, in the specific case of the Klein bottle, I started looking for a nonsingular immersion in its classical shape and in a single piece because I needed a 3D model suitable to be used with a 3D printing system to be built as a real object. Later I learned that some conditions were not necessary, but the work was done by then!

\section{A History of the Klein Bottle Representations}

Until the 1970s, geometry and topology textbooks showed handmade drawings of the Klein bottle, which strictly followed the original geometric construction given by Klein, i.e., a tube that penetrates itself [15]. A second version of the Klein bottle, obtained by moving a lemniscate around a circle while it rotates, in its plane, half a turn around its center (see Figure 3), appeared in 1976 in a paper by $\mathrm{T}$. Banchoff [3] dealing with minimal submanifolds of the bicylinder boundary. The author claims that he first discovered the model on the three-sphere, then projected it in three-space.
The first computer graphics of this version of the Klein bottle appeared in 1982 in a work by S. Feiner, D. Salesin, and T. Banchoff as a case study for an animation language [4]. The paper provides wireframe and rendered images of the surface and also shows a sequence of steps of its geometrical construction which helps in the understanding of the topological structure. In this version, often called the figure-eight bottle or the Banchoff bottle, the self-intersection curve has a neighborhood made of two Möbius strips, while in the classical model the neighborhood of the double curve is a couple of annuli. Another interesting model of the bottle, very beautiful but maybe less immediately understood with respect to the previous one, can be obtained by projecting stereographically to $\mathbb{R}^{3}$ a minimal surface that lives on the three-sphere $\mathbb{S}^{3}$ and that belongs to one of the families of the minimal surfaces classified by H. B. Lawson in [19]. Lawson's work, dated 1970 , does not contain any attempt at visualizing the surface. Its projection to $\mathbb{R}^{3}$ is a family of circles in space but also the zero set of a polynomial of degree six. In F. Apéry's book Models of the Real Projective Plane [1] one can find both parametric and algebraic formulas for the surface and-to my knowledge-the first computer graphics of it. In 1991, S. Dickson [11] provided equations for the bottle in its classical shape. He parameterized the surface in two distinct parts, whose union gives the desired object. This version was included among the examples in release 2.2 of the software Mathematica [25], a system for doing mathematics by computer that contains a powerful visualization tool for geometric objects. Again in 1991, D. Cox, G. Francis, and R. Idaszak made a striking video titled The Etruscan Venus [14], which shows a morphing (homotopy) between two new versions of the Klein bottle, one as the connected sum ${ }^{1}$ of two copies of the Steiner Roman surface, the other as the connected sum of two copies of the Boy surface (Figure 2). The name is due to the fact that the double Roman surface recalls the shape of a woman's body. This morphing was obtained by modifying another remarkable one, called Romboy, which leads from the Roman surface to the Boy surface, worked out by F. Apéry and described and illustrated in [1]. A bottle composed of four pieces was worked out in 1993 by P. Chang, at that time a student at the Department of Mathematics at UCLA, and illustrated by S. Dickson [10]. The resulting model is suitable to be designed with $\mathrm{CAD}^{2}$ software, even

\footnotetext{
${ }^{1}$ The connected sum of two closed surfaces is obtained by removing a small disk from each one and by smoothly gluing together the borders of the holes; see also the section about regular homotopy classes of immersed surfaces.

${ }^{2}$ Computer Aided Design.
} 

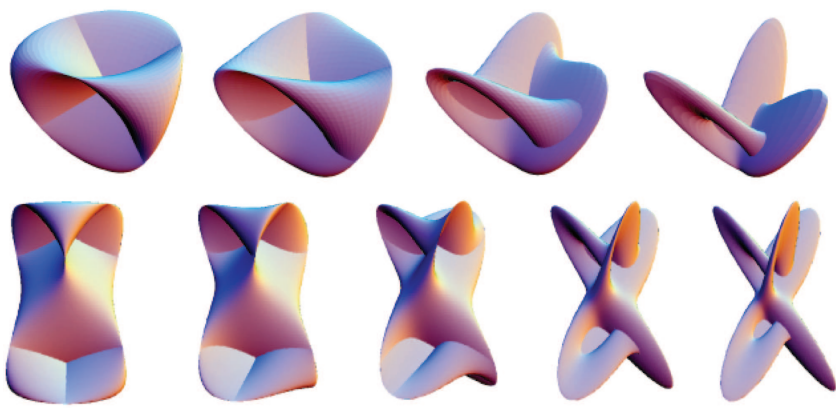

Figure 2. Top sequence: four steps of the Romboy morphing ( $F$. Apéry); bottom sequence: five steps of the Etruscan Venus morphing (D. Cox, G. Francis, R. Idaszak).

by someone who knows very little mathematics, as it is the union of a set of sweepings of circular arcs along other circular arcs. In 1994, D. Cervone, in answer to a question posed by T. Banchoff [6], produced an effective representation of the Klein bottle in its classical shape. After that, J. Beall added transparency to the object and animated it. The resulting short movie was presented to D. Struik on his $100^{\text {th }}$ birthday [16]. The surface is composed of two pieces, a tube around half of a lemniscate together with a piece of surface of revolution generated by the second half of the same lemniscate (more details follow later). A significant new step about representations of the Klein bottle was performed in 1999 by M. Trott [24], who provided both an algebraic and a parameterized expression of the surface in its classical shape, in a single piece, and used it as a test case to explain a survey of features of Mathematica software.

\section{Regular Homotopy Classes of Immersed Surfaces}

How much different from each other are the Klein bottles described in the previous section, apart from their appearance? The notion of regular homotopy of immersions gives us a rigorous criterion for telling whether any two immersions of a surface are essentially the same immersion or not. This is a quite technical subject, from which we will get only the key ideas that apply to our context. For a detailed discussion on the subject see for example [21]. A homotopy between two immersions is a continuous deformation which has as initial stage the first immersion and as final stage the second immersion; the homotopy is an equivalence relation; a regular homotopy is a smooth homotopy that is an immersion at each stage. An immersed surface can be considered as an equivalence class of immersions of a surface up to regular homotopy. It is possible to define a small set of immersed surfaces which generate, by means of the connected sum \#, every compact immersed
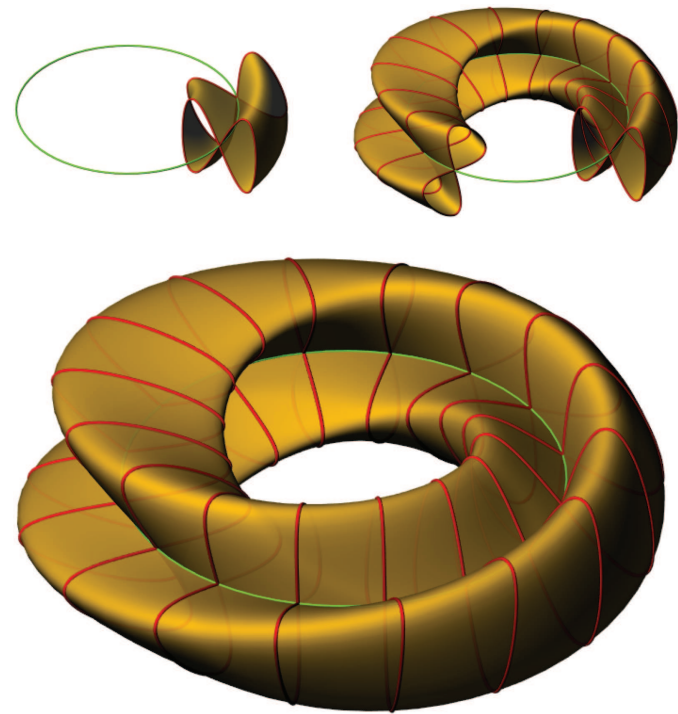

Figure 3. Three views of a Klein bottle generated by moving a lemniscate.

surface in $\mathbb{R}^{3}$. In particular, every immersion of the Klein bottle must be regularly homotopic to a connected sum of two copies of the Boy surface, which comes in two different classes, $B$ and its mirror image $\bar{B}$ (see [1] for a detailed discussion about the Boy surface). Taking off a disk from $B$ or $\bar{B}$ gives us respectively a right- or a left-handed Möbius band.

With this said, we can immediately recognize the regular homotopy classes of all the immersions of the Klein bottle described in the previous section: the classical one belongs to the $B \# \bar{B}$ class, for it can be seen as the union of two copies of the Möbius band which are the mirror images of each other, so they are twisted in opposite senses. Banchoff's bottle comes in two different classes, $B \# B$ and $\bar{B} \# \bar{B}$, because it is made of two Möbius bands twisted in the same sense. Lawson's bottle also contains two copies of the Möbius band twisted in the same sense; thus it comes in the same two classes as Banchoff's. The Etruscan Venus is nothing more than a canonical representative of the classes $B \# B$ and $\bar{B} \# \bar{B}$, depending on the "chirality" of the generators chosen. Are the three classes described so far all the possible ones? No. According to a result by James and Thomas [17] there must be four such classes, the number of classes given by $2^{2-x}$ where $x$ is the Euler characteristic of the surface, which is zero for the Klein bottle. This implies that a fourth kind of bottle, not equivalent to the three already mentioned, must exist. An interesting work concerning this aspect is being done at the moment by C. H. Séquin [23]. The author investigates how to construct a good representative for the fourth class and, along the way, shows several examples of immersions of 

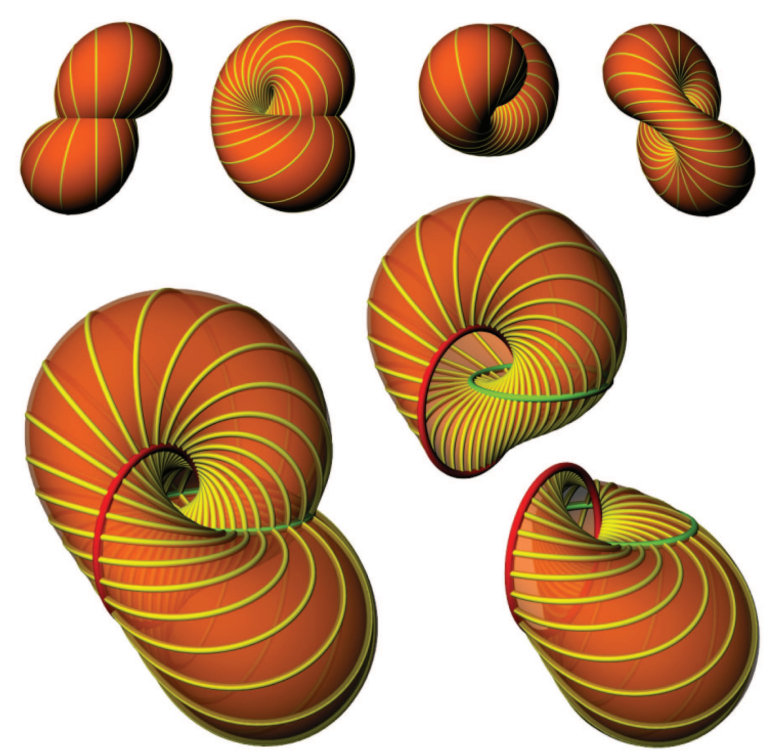

Figure 4. The Klein bottle as a one-parameter family of circles (H. B. Lawson): the top sequence shows four coordinate views; the larger images show that the surface is the union of two Möbius bands glued along their circular borders, colored in red, which pass through each other along the green circle. The red and green circles lie on the surface but do not belong to the family that generates the surface itself; both meet the generating family orthogonally.

the Klein bottle, some of which have never been depicted before.

\section{Simple Equations for the Klein Bottle}

This section recalls the parametrization and the resulting graphic images of two of the immersions of $\mathbb{K}$ in $\mathbb{R}^{3}$ which we called canonical at the beginning of the article, due respectively to T. Banchoff and to B. Lawson:

BanchoffBottle $(u, v)$ :

(1)

$\left\{\begin{array}{l}x=\left(a+\cos \left(\frac{u}{2}\right) \sin v-\sin \left(\frac{u}{2}\right) \sin (2 v) \cos u,\right. \\ y=\left(a+\cos \left(\frac{u}{2}\right) \sin v-\sin \left(\frac{u}{2}\right) \sin (2 v) \sin u,\right. \\ z=\sin \left(\frac{u}{2}\right) \sin v+\cos \left(\frac{u}{2}\right) \sin (2 v),\end{array}\right.$

LawsonBottle $(u, v)$ :

(2)

$$
\left\{\begin{array}{l}
x=\cos 2 u \sin v / \\
(1-(\sin u \cos u+\sin 2 u \sin v) / \sqrt{2}), \\
y=(\sin 2 u \sin v-\sin u \cos v) / \\
\sqrt{2}(1-(\sin u \cos u+\sin 2 u \sin v) / \sqrt{2}), \\
z=\cos u \cos v / \\
(1-(\sin u \cos u+\sin 2 u \sin v) / \sqrt{2}) .
\end{array}\right.
$$

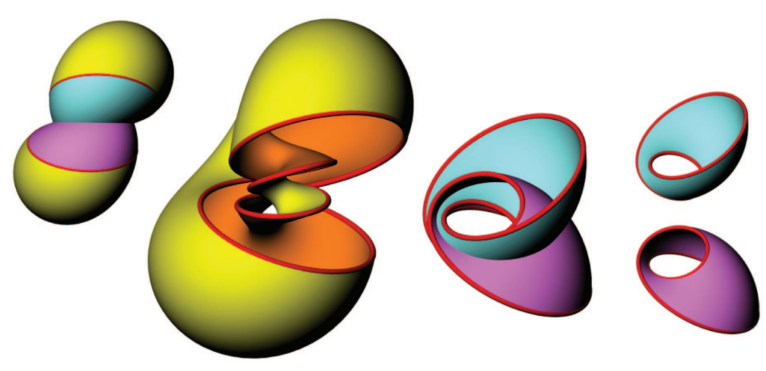

Figure 5. The same Klein bottle of previous image, cut in a different way. The part around the self-intersection circle is made of two Möbius bands. Cutting them away, the remaining portion of the surface is orientable, so it is possible to give its faces two different colors.

\section{Some Classical-Looking Klein Bottles}

In this section we recall some formulas and images of the Klein bottle in its classical shape, already mentioned in the chronological list. Some of them give the desired surface as the union of two or more pieces; some others have complicated expression, far from the shortness and the elegance of (1) and (2). The first one was created in 1994 by D. Cervone in answer to a scenario proposed by $\mathrm{T}$. Banchoff: take a Bernoulli lemniscate and use half of it as the directrix for a tube and the other half as the generatrix for a revolution surface. Figure 6 shows how the construction is carried out. The union of the two pieces, which meet tangentwise, is a very effective and beautiful model of the bottle and fits perfectly with the description given by Klein. Its parametrization is also very simple. Now, before going on with the other two models of the classical bottle, due to S. Dickson and M. Trott, respectively, we will recall the basic technique of generating a tube around a curve. Let $\alpha(t)=(x(t), y(t)), t \in[a, b]$, be a curve lying on the $x y$-plane satisfying $\left\|\alpha^{\prime}(t)\right\| \neq 0$. Let $\mathbf{k}=(0,0,1)$ be the $z$-axis unit vector and $\mathbf{T}=\frac{\alpha^{\prime}}{\left\|\alpha^{\prime}\right\|}$ be the unit tangent vector field of $\alpha(t)$. Let $\mathbf{N}=\mathbf{k} \wedge \mathbf{T}$. Then the couple of unit vectors $(\mathbf{N}, \mathbf{k})$ is a moving frame orthogonal to $\alpha^{\prime}(t)$ and can be used to construct a tube around $\alpha(t)$ as follows:

(3)

$$
\begin{array}{r}
\operatorname{tube}(t, \theta)=\alpha(t)+r(t)(\cos \theta \mathbf{N}+\sin \theta \mathbf{k}), \\
(t, \theta) \in[a, b] \times[0,2 \pi],
\end{array}
$$

where the scalar continuous function $r(t)$ gives the radius of the tube. Note that, in this definition, $\mathrm{N}$ is not the standard unit normal to the curve $\alpha(t)$, which would point to the center of the osculating circle of $\alpha(t)$, because it would not be defined at the points where the curvature of $\alpha(t)$ vanishes.

The first model of the Klein bottle as a tube around a curve (in fact, the union of two distinct 


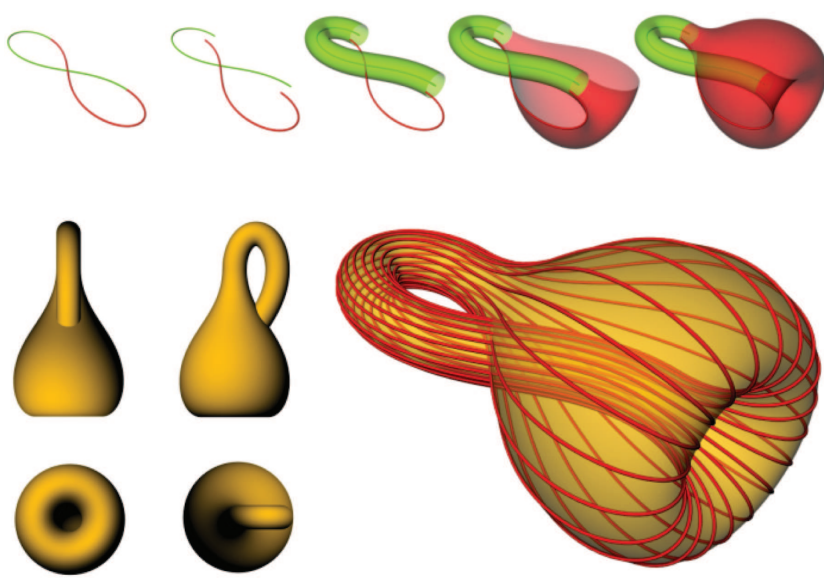

Figure 6. Construction of the Banchoff-Cervone bottle. Top sequence: a Bernoulli lemniscate is cut in two halves, one of which is moved aside by a distance $r$; a tube of constant radius $r$ is generated about one of the halves and then a surface of revolution is generated by making the second half of the lemniscate turn around the axis passing by the two ends of the first half. Bottom: coordinate views and perspective of the resulting object.

tubes) is due to S. Dickson [11]. It is built up according to the scheme just described, except for the choice of the moving pair of vector fields, which is not orthogonal to the curve. Here is its parametrization:

(4)

$$
\begin{aligned}
& \text { DicksonBottle }(u, v) \\
& x=\left\{\begin{array}{r}
\left\{\begin{array}{l}
6 \cos u(1+\sin u)+4\left(1-\frac{1}{2} \cos u\right) \cos u \cos v \\
\text { for } 0 \leq u \leq \pi, \\
6 \cos u(1+\sin u)+4\left(1-\frac{1}{2} \cos u\right) \cos (v+\pi) \\
\text { for } \pi<u \leq 2 \pi
\end{array}\right. \\
y=\left\{\begin{array}{l}
16 \sin u+4\left(1-\frac{1}{2} \cos u\right) \sin u \cos v \\
16 \sin u \quad \text { for } \pi<u \leq 2 \pi,
\end{array}\right. \\
z=4\left(1-\frac{1}{2} \cos u\right) \sin v .
\end{array}\right.
\end{aligned}
$$

Parametrization (4) defines two distinct tubes, the first one built up on a frame which moves along the central curve remaining parallel to the $x z$-plane, the second one connecting the two ends of the first tube through a rotation of $\pi$ of the moving frame. The union of these two parts turns out to be a good-looking object (Figure 7), which renders properly the idea outlined by Klein.

Note that the central curve used in this construction

$$
\alpha(t):\left\{\begin{array}{l}
x=6 \cos t(1+\sin t), \\
y=16 \sin t
\end{array}\right.
$$
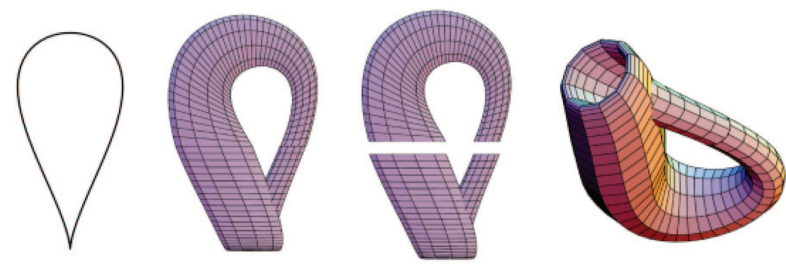

Figure 7. Klein bottle according to S. Dickson's definition; on the left, the central curve. Image on the right: courtesy of Wolfram Research, Inc.

is a piriform, a well-known curve (see for example [8] or [9]) whose general parametrization is

$$
\text { (6) } \operatorname{Piriform}(t):\left\{\begin{array}{l}
x=a(1+\sin t), \\
y=b \cos t(1+\sin t) \text {. }
\end{array}\right.
$$

It can be easily proven, and also guessed by looking at Figure 7, that (4) defines a singular immersion, as the two tubes do not meet tangentwise along the common boundaries, and, of course, it would be better to get the surface as the image of a single parametrization, with no use of inequalities, as in (4). This has been achieved by M. Trott, who defines a parameterized bottle as a midstep in order to get an algebraic definition of it via Mathematica. We are interested in his parametric definition, which closely follows the scheme defined by (3). Trott puts some constraints on the directrix and on the radius, which can be summarized in the following:

$$
\begin{array}{ll}
\text { i) } & \alpha(a)=\alpha(b), \\
\text { ii) } & \alpha^{\prime}(a)=-\alpha^{\prime}(b), \\
\text { iii) } & r(a)=r(b), \\
\text { iv) } & r^{\prime}(a)=r^{\prime}(b)= \pm \infty .
\end{array}
$$

Conditions i), ii), and iii) mean that the two ends of the tube must be coincident, while iv) means that they must meet tangentwise. The curve and the radius he uses are

(8)

$$
\begin{aligned}
& \beta(t)=\left(\frac{1}{t^{4}+1}, \frac{t^{2}+t+1}{t^{4}+1}\right), \quad t \in(-\infty,+\infty), \\
& r(t)=\frac{84 t^{4}+56 t^{3}+21 t^{2}+21 t+24}{672\left(1+t^{4}\right)}
\end{aligned}
$$

and the resulting image is shown in Figure 8. Equations (8) define an immersion, but the resulting shape is somehow edgy because of the choice of a directrix whose curvature has a nonsmooth behavior. Moreover, as $t$ ranges on an open interval, when one tries to plot the surface, there is a missing strip corresponding to a neighborhood of the cusp (Trott uses $t \in[-20,+20]$ in his plots). 


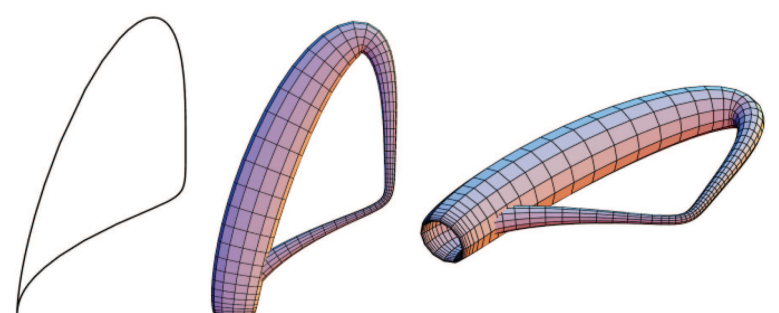

Figure 8 . Klein bottle according to M. Trott's definition; on the left, the central curve.

\section{A New Description}

We propose here a new parametrization of the bottle in its classical shape. Starting from the two constructions described in the previous paragraph, it is natural to try to construct a new surface by taking the best features from both: the beautiful and symmetric directrix of Dickson's version and the rigorous geometric scheme of Trott's. In order to use the piriform as a directrix for our tube, we reparameterize it to make it start and end at the cusp:

(9) $\quad \gamma(t)=\left\{\begin{array}{l}a(1-\cos t), \\ b \sin t(1-\cos t),\end{array} \quad t \in(0,2 \pi)\right.$.

A suitable radius, which satisfies iii) and iv) of (7), is, for example,

$$
r(t)=c-d(t-\pi) \sqrt{t(2 \pi-t)} .
$$

Parameters $c$ and $d$ affect, respectively, the radius of the whole tube and the difference between its minimum and maximum value. The resulting plot, with $(a, b, c, d)=\left(20,8, \frac{11}{2}, \frac{2}{5}\right)$ and $(t, \theta) \in(0,2 \pi) \times[0,2 \pi]$, is shown in Figure 9 .

\section{Some Remarks}

Although, in our opinion, the described result is rather satisfactory, there are some facts to be pointed out. First, the parametrization of our surface, extensively written, has a long and complicated expressions. Secondly, similarly to what happens with Trott's parametrization, the image of the immersion fails to be closed because it misses a circle at the cusp of the directrix, as $\left\|\gamma^{\prime}\right\|$ vanishes at $t=0$ and $t=\pi$, while the scheme used needs $\left\|\gamma^{\prime}\right\|$ to be nonzero everywhere. A way to eliminate this issue is to use one-half of the Dumbbell curve (see [9]) as a directrix. This is a famous sextic curve which also has the following parametrization:

(11)

$$
\text { dumbbell }(t):\left\{\begin{array}{l}
x=\sin t, \\
y=\sin ^{2} t \cos t,
\end{array} \quad t \in[0,2 \pi] .\right.
$$

If $t$ ranges in $I=[0, \pi]$, one obtains a curve that satisfies the first three conditions of (7) and whose tangent vector is well defined for all $t \in I$,
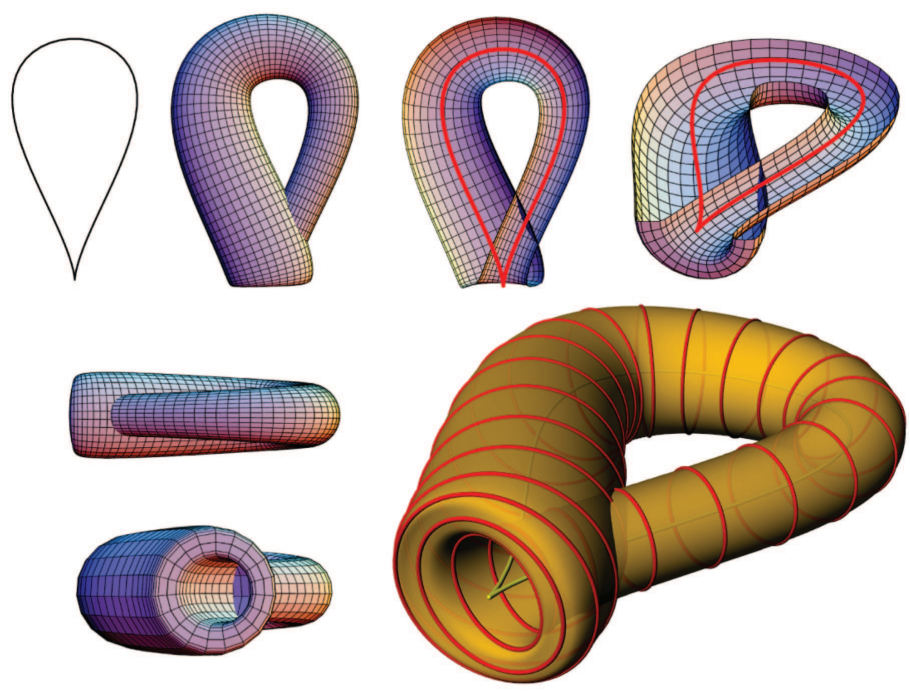

Figure 9. The piriform curve and a tube around it: an immersion of the Klein bottle in $\mathbb{R}^{3}$.

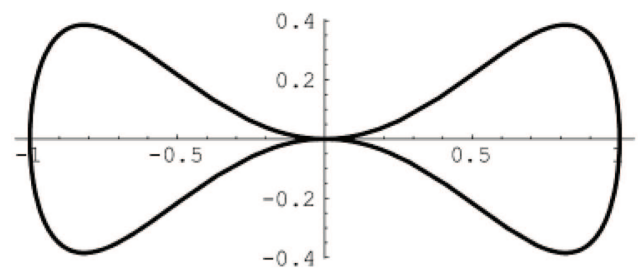

Figure 10. Dumbbell curve.
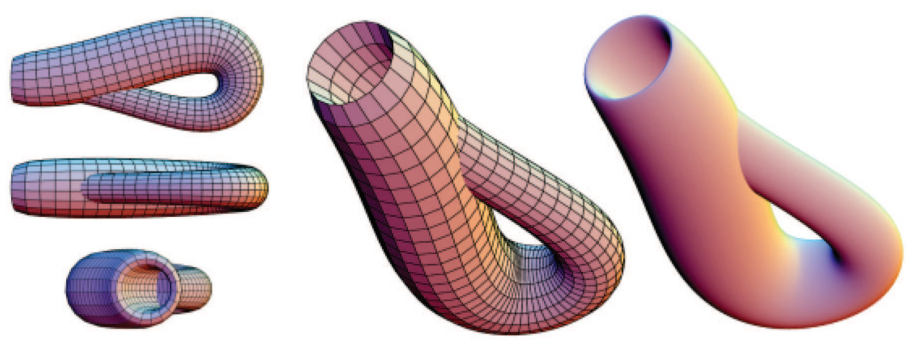

Figure 11. Some views of the Klein bottle as a tube around Dumbbell curve.

so it is possible to use a closed rectangle as a domain for the immersion, obtaining a closed image. By using a stretched Dumbbell curve $\alpha(t)=$ $\left(5 \sin t, 2 \sin ^{2} t \cos t\right)$ as the directrix and $r(t)=$ $\frac{1}{2}-\frac{1}{30}(2 t-\pi) \sqrt{2 t(2 \pi-2 t)}$ as the radius function, with $t \in[0, \pi]$, we obtain another example of (closed) immersion of the Klein bottle (Figure 11).

\section{Conclusion}

After collecting some of the most interesting representations of the Klein bottle as a surface immersed in $\mathbb{R}^{3}$, recalling their equations and showing their graphics, we define two new 

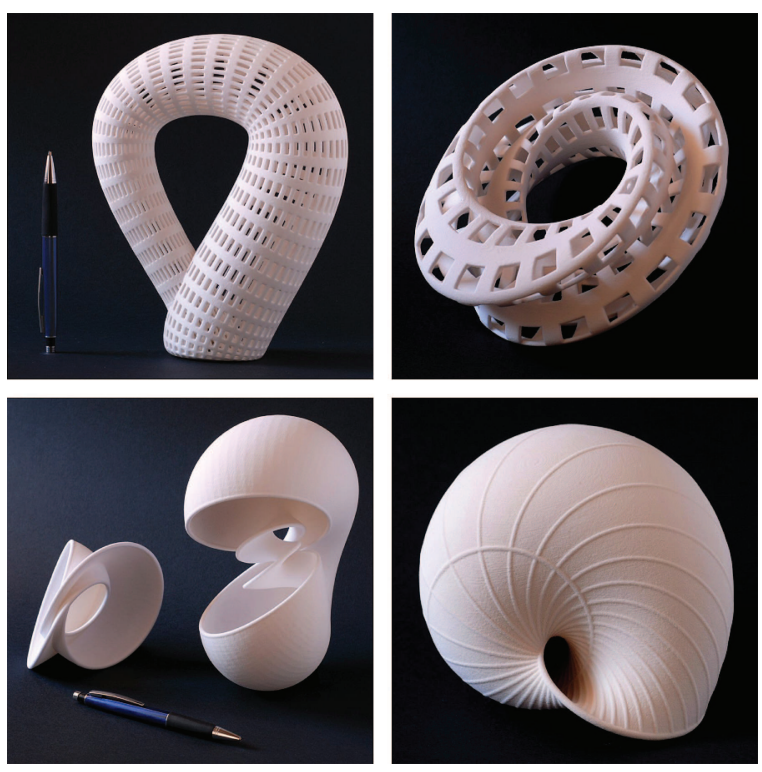

Figure 12. Photos of 3D printed models of three versions of the Klein bottle made by a Z-Printer Z310 Plus (material: plaster). Top left: Klein bottle according to parametrization (9)-(10); top right: Klein bottle according to parametrization

(1); bottom left: Klein bottle cut in two parts, according to parametrization (2); bottom right: one half of the same surface, cut in such a way that each of the two halves is a Möbius band with circular border; some circular arcs lying on the surface itself are highlighted. (Models and pictures were created by the author.)

immersions of the bottle in the shape outlined by Klein in 1882, with a reasonably good appearance. They are suitable to make computer plots and, after generating a solid shell around them, to be used as an input dataset on 3D printing systems. The mathematical expression of both is still complicated and far from the elegance of versions like (1) and (2). They are intended to be a midstep towards an immersion of the Klein bottle in $\mathbb{R}^{3}$ which we would like to call canonical from both a mathematical and a historical point of view.

\section{Acknowledgments}

The author wishes to thank F. Apéry, T. Banchoff, S. P. Dickson, G. K. Francis, and C. H. Séquin for helpful discussions, as well as a cast of referees for the article.

The graphics in this article were produced with Mathematica by Wolfram Research, Inc.; some of the 3-D data was treated and rendered with McNeel Rhinoceros, v.4.

\section{References}

[1] F. APÉRY, Models of the Real Projective Plane, Vieweg, 1987.
[2] F. APÉRY and G. FrAnzoni, Il rovesciamento della sfera: un modello materiale della fase centrale, Rendiconti del Seminario della Facoltà di Scienze dell'Università di Cagliari (1999), 1-18.

[3] T. BANCHOFF, Minimal submanifolds of the bicylinder boundary, Boletim da Sociedade Brasileira de Matematica 7 (1976), 37-57.

[4] Steven Feiner, David SAlesin, and Thomas BanCHOFF, Dial: A Diagrammatic Animation Language, IEEE Computer Graphics and Applications, September (1982), 43-54.

[5] T. Banchoff, Beyond the Third Dimension: Geometry, Computer Graphics and Higher Dimensions, Second Edition, Freeman, 1990.

[6] _ private communication.

[7] C. P. Bruter, Mathematics and Art, Springer, Paris, 2002.

[8] R. CADDEO and A. GRAY, Curve e Superfici, Volume I, CUEC, 2000.

[9] Concise Encyclopedia of Mathematics, Second Edition, CRC Press LLC, 2003.

[10] P. Chang, Klein bottle in four parts, 1993, http:// www. ifp.il1inois.edu/ sdickson/K1ein/K1ein. html.

[11] S. Dickson, Klein bottle graphic, 1991, http:// 1ibrary.wolfram.com/infocenter/MathSource/ 4560/

[12] IVARS Peterson, Plastic Math, Science News, vol. 140, no. 5, Aug. 3, 1991, 72-73.

[13] G. FISCHER, Mathematische Modelle: Mathematical Models, Vieweg, Inc., 1986.

[14] G. K. Francis, A topological picturebook, SpringerVerlag, New York, 1987.

[15] D. Hilbert and S. Cohn-Vossen, Geometry and the Imagination, Chelsea Publishing Co., New York, 1952.

[16] A. JaCkson, Dirk Struik celebrates his 100th, Notices of the AMS 42 (1995), 43-45.

[17] I. JAMES and E. THOMAS, Note on the classification of cross-sections, Topology 4 (1966), 351-359.

[18] F. KLEIN, Über Riemann's theorie der algebraischen Funktionen und ihrer Integrale, Teubner Verlag, Leipzig, 1882.

[19] H. B. LAwSON, Complete minimal surfaces in $\mathbb{S}^{3}$, Ann. of Math. 92 (1970), 335-374.

[20] R. S. PALAIS, The visualization of mathematics: Towards a mathematical exploratorium, Notices of the AMS 46 (1999), 647-658.

[21] U. PINKALL, Regular homotopy classes of immersed surfaces, Topology 24 (1985), 421-432.

[22] C. H. SÉQUIN, Art, math, and computers: New ways of creating pleasing shapes, Bridges 1998, Mathematical Connections in Art, Music, and Science, Reza Sarhangi, Editor, 1998, 1-10.

[23] _ From Möbius bands to Klein-Knottles, to appear in the Proceedings of the Bridges Conference, 2012.

[24] M. Trotт, Constructing an algebraic Klein bottle, Mathematica in Education and Research 8 (1999), 24-27.

[25] http://www. wolfram.com

[26] http://www. zcorp.com 